\title{
CONSTRUÇÃO DA PERMANÊNCIA DO OBJETO EM CRIANÇAS NASCIDAS PRÉ-TERMO MUITO BAIXO PESO
}

\author{
Object permanence construction in very low birth weight \\ preterm infants
}

\author{
Karina Elena Bernardis Bühler ${ }^{(1)}$, Fabíola Custódio Flabiano ${ }^{(2)}$, \\ Amanda Elias Mendes ${ }^{(3)}$, Suelly Cecilia Olivan Limongi ${ }^{(4)}$
}

\begin{abstract}
RESUMO
Objetivo: descrever e comparar o processo de construção da permanência do objeto entre crianças com histórico de prematuridade e muito baixo peso e crianças nascidas a termo com desenvolvimento típico. Métodos: participaram deste estudo 22 crianças, sendo 8 crianças nascidas prétermo e muito baixo peso (grupo pesquisa - GP) e 14 crianças nascidas a termo (grupo controle GC). Os sujeitos foram submetidos a avaliações mensais de cognição e linguagem, contendo a avaliação da noção de permanência do objeto. Todas as sessões foram registradas em vídeo e transcritas em protocolo específico. As crianças de ambos os grupos foram avaliadas dos 6 aos 12 meses, sendo considerada para o GP, a idade corrigida. Os dados foram analisados de forma quantitativa e qualitativa. Resultados: observou-se diferença estatisticamente significante entre os dois grupos para todas as fases da construção da permanência do objeto, evidenciando o atraso (uma média de 2 a 3 meses de atraso) no desenvolvimento cognitivo, apresentado pelo GP, em relação ao GC, durante o período sensório-motor. Conclusão: os resultados reforçam a idéia de que as crianças nascidas pré-termo e muito baixo peso apresentam risco para alterações ou atrasos no desenvolvimento cognitivo e de linguagem, justificando a elaboração de programas de intervenção fonoaudiológica junto a essas crianças, já durante o primeiro ano de vida.
\end{abstract}

DESCRITORES: Desenvolvimento Infantil; Cognição; Prematuro; Lactente

\section{INTRODUÇÃO}

Os avanços científicos e tecnológicos da medicina obstétrica e perinatal aumentaram drasticamente as taxas de sobrevivência de recém-nascidos de muito

(1) Fonoaudióloga do Hospital Universitário da Universidade de São Paulo; Doutoranda pelo Programa de Pós-Graduação em Ciências da Reabilitação da Faculdade de Medicina da Universidade de São Paulo.

(2) Fonoaudióloga Pesquisadora do Laboratório de Investigação Fonoaudiológica em Síndromes e Alterações Sensório-Motoras da Faculdade de Medicina da Universidade de São Paulo; Doutoranda pelo Programa de Pós-Graduação em Ciências da Reabilitação da Faculdade de Medicina da Universidade de São Paulo.

(3) Aluna de Graduação em Fonoaudiologia pela Faculdade de Medicina da Universidade de São Paulo.

(4) Fonoaudióloga; Professora Livre Docente do Curso de Fonoaudiologia da Faculdade de Medicina da Universidade de São Paulo. baixo peso (peso de nascimento inferior a 1500g) nas duas últimas décadas. Nesse sentido, ao longo do tempo, as preocupações não foram mais dirigidas à mortalidade, mas redirecionadas às morbidades associadas à prematuridade e à qualidade de vida desses bebês ${ }^{1,2}$.

Uma das principais finalidades do acompanhamento dos bebês prematuros de muito baixo peso (PTMBP) é a identificação precoce de sinais de anormalidades do desenvolvimento para que uma intervenção apropriada possa ter início, na tentativa de minimizar estas alterações e integrar essas crianças no seio da sociedade ${ }^{3}$.

O prognóstico do desenvolvimento das crianças nascidas PTMBP depende da complexa interação de fatores biológicos, sociais e ambientais atuantes no cérebro imaturo e vulnerável dessas crianças. Considerando que o primeiro ano de vida é extremamente importante para a maturação neurológica, formação do vínculo afetivo e construção do conhecimento, as crianças com histórico de prematuridade e muito baixo peso 
apresentam alto risco para alterações nos desenvolvimentos cognitivo e de linguagem. Crianças pré-termo têm sido consistentemente identificadas como crianças que apresentam problemas de linguagem, tanto receptiva quanto expressiva, especialmente durante os anos pré-escolares ${ }^{4}$ e escolares ${ }^{5-7}$. Presença de alterações articulatórias e de fluência, déficits de vocabulário, sistema lexical, sintático, gramatical e discriminação auditiva também são mais comuns entre crianças pré-termo do que as nascidas a termo ${ }^{5,8-13}$. Em função disso, algumas pesquisas nacionais têm apontado para a importância do acompanhamento dessas crianças, principalmente nas questões relacionadas à linguagem e à cognição ${ }^{14-16}$.

Estudos longitudinais têm mostrado que $50 \%$ a $70 \%$ das crianças nascidas pré-termo e extremo baixo-peso, mesmo na ausência de patologias de base, apresentam dificuldades de aprendizagem, desordem do déficit de atenção e hiperatividade, neuropsicológicos específicos e problemas comportamentais ${ }^{17-21}$. Alguns estudos têm apontado ainda para atraso na aquisição de algumas funções da linguagem e alterações de memória ${ }^{22-25}$, enquanto outros têm sugerido que, mesmo em crianças com baixo risco para alterações neurológicas, podem ser observados déficits cognitivos específicos decorrentes da prematuridade ${ }^{12,26,27}$.

Estudo realizado nos Estados Unidos ${ }^{28}$ com o objetivo de verificar se recém-nascidos PTBMP sem alterações neurológicas importantes apresentavam padrões de desenvolvimento similares aos de crianças nascidas a termo, aos três e cinco anos, demonstrou que, mesmo considerando a idade corrigida, 84\% das crianças PTMBP apresentaram desempenho inferior nos testes cognitivos e alterações de linguagem, quando comparadas às crianças nascidas a termo.

Os resultados de uma pesquisa realizada em instituição internacional ${ }^{29}$ mostraram que crianças nascidas prematuramente sem maiores déficits neurológicos e com nível cognitivo normal podem apresentar dificuldades específicas em atenção sustentada, processamento visuo-espacial e memória de trabalho espacial quando avaliadas nas idades de três a quatro anos.

Autores de outro estudo ${ }^{13}$ afirmam que as dificuldades iniciais no processamento auditivo e lingüístico, devido à imaturidade do sistema nervoso central (SNC) e estímulo externo inadequado, podem causar um atraso ou um padrão diferente para o desenvolvimento da percepção de fala e discriminação, o qual pode afetar as competências lingüísticas tardias, como a compreensão e produção.

Quanto menores o peso ao nascimento e a idade gestacional, maior a probabilidade de altera- ções do desenvolvimento da linguagem, as quais podem persistir até a idade escolar e comprometer o desempenho da criança ${ }^{7,13,30-32}$

Os déficits que as crianças nascidas PTMBP podem apresentar são considerados como conseqüência de complicações médicas associadas com nascimento prematuro, tais como: hipóxia, hemorragia intraventricular, alterações metabólicas e nutricionais, estresse biológico e ambiental, o que pode causar anormalidades cerebrais ${ }^{29}$, as quais têm sido relatadas por alguns estudos com neuroimagens ${ }^{33}$. Porém, embora a associação entre anormalidades cerebrais e prejuízos cognitivos seja observada em crianças pré-termo, esta relação ainda não está bem definida ${ }^{34}$.

A construção da noção de permanência do objeto, de acordo com o modelo proposto pela Epistemologia Genética, tem início por volta do $8^{\circ}$ mês de vida e é de fundamental importância, visto que leva à formação da imagem mental, condição premente à constituição da representação que, por sua vez, favorece o desenvolvimento da linguagem oral ${ }^{35}$.

Autores ${ }^{36}$ consideram que a construção do esquema do objeto permanente é solidária com a organização do universo prático e com a estruturação causal. Esse processo é progressivo e evolui conforme a construção do esquema de objeto permanente, conduzindo à percepção de mudança de estado, posição ou deslocamentos consecutivos no espaço. A imagem mental permite a evocação de objetos, das ações e das situações ausentes, sendo uma representação figurativa de realidades vividas, que está em franco desenvolvimento durante o $6^{\circ}$ estágio do período sensório-motor ${ }^{37}$.

Sendo a constituição da noção de permanência do objeto uma construção cognitiva fundamental ao desenvolvimento da linguagem, visto que levará à formação da imagem mental e à representação e, sabendo-se que as crianças nascidas PTMBP apresentam risco para alterações de linguagem e cognição, a hipótese do presente trabalho consistiu da idéia de que seria possível observar diferenças entre crianças nascidas pré-termo e a termo em relação à construção da noção de permanência do objeto, já no primeiro ano de vida.

Portanto, o objetivo deste estudo foi descrever e comparar o processo de construção da noção de permanência do objeto entre crianças com histórico de prematuridade e muito baixo peso e crianças nascidas a termo com desenvolvimento típico.

\section{MÉTODOS}

O presente estudo compreendeu um grupo pesquisa (GP) e um grupo controle (GC). O GP foi com- 
posto por 8 crianças nascidas pré-termo com peso de nascimento inferior a $1500 \mathrm{~g}$ e idade gestacional inferior a 34 semanas, nascidas no Hospital Universitário da Universidade de São Paulo, participantes do Ambulatório de Seguimento de Recém-nascidos de Alto Risco do Hospital Universitário. Este Ambulatório conta com uma equipe multiprofissional composta por pediatra, neurologista, otorrinolaringologista, oftalmologista, fonoaudiólogo, nutricionista, enfermeiro e fisioterapeuta, que realizam o acompanhamento mensal dessas crianças durante o primeiro ano de vida. A idade gestacional do GP foi calculada com base na data da última menstruação (DUM) (informação esta obtida a partir do prontuário médico da criança).

O grupo controle (GC) foi constituído por 14 crianças nascidas a termo no Hospital Universitário, saudáveis, sem intercorrências pré, peri ou pós-natais, adequadas para idade gestacional e com peso de nascimento superior a $2500 \mathrm{~g}$ e inferior a $3999 \mathrm{~g}$. As crianças do GC eram todas acompanhadas no Ambulatório de Puericultura e/ou pertencentes à creche do Hospital Universitário.

Foram admitidos como critérios de exclusão para o GP a presença de má-formações, síndromes genéticas e asfixia neonatal grave. Os critérios de inclusão para ambos os grupos envolviam acompanhamento pediátrico e otorrinolaringológico, boas condições de saúde e audição normal. Para tanto, todas as crianças foram submetidas à avaliação audiológica composta por otoscopia, timpanometria, pesquisa de reflexos acústicos, emissões otoacústicas tran-sientes (EOAT) e Audiometria de Tronco Encefálico (ABR).

Os sujeitos foram submetidos a avaliações mensais de cognição, as quais foram registradas em vídeos e transcritas em protocolo específico. Para tanto, foi utilizado o Protocolo de Observação do Desenvolvimento Cognitivo e da Linguagem Expressiva, elaborado com base nos estudos de Piaget ${ }^{35,38,39}$, Bates ${ }^{40}$, Iverson et al ${ }^{41}$, Capone e McGregor ${ }^{42}$, que contém, entre outros itens, a noção de permanência do objeto. Todas as crianças foram avaliadas dos seis aos 12 meses, sendo considerada para o GP, a idade corrigida (calculada subtraindo-se a idade gestacional de 40 semanas, e subtraindo-se novamente esta diferença da idade cronológica no momento das avaliações).

As faixas etárias foram divididas em quinzenas, visando a redução de possíveis diferenças entre as filmagens que fossem realizadas no início ou final do mês, em relação à data de nascimento da criança.

Foram consideradas crianças de ambos os sexos, visto que esta variável não interfere nos resulta- dos deste estudo.

O material utilizado foi composto por dois lenços de cores diferentes, uma bola, um chocalho, um bicho de borracha e uma caixa pequena de fósforos com clipes dentro.

Os lenços eram dispostos em posições diferentes em frente à criança e o objeto de interesse era, então, totalmente escondido sob um deles, às vistas da mesma. Caso a criança não demonstrasse intenção de puxar o lenço para pegar o objeto, o examinador deixava uma pequena parte do objeto descoberto. Caso a criança fosse capaz de procurar pelo objeto totalmente escondido, o mesmo era, então, deslocado para o outro pano, às vistas da criança. Se a criança realizasse a tarefa com sucesso, o examinador, então, realizava o deslocamento invisível do objeto, escondendo-o primeiramente dentro de sua mão e, em seguida, deslocando-o para debaixo do colchão ou para dentro do bolso do jaleco. O procedimento foi aplicado por três vezes consecutivas, sendo considerada e pontuada a resposta de maior ocorrência. Foram considerados 0 ponto para não procura; 1 ponto para procura do objeto parcialmente escondido; 2 pontos para procura do objeto totalmente escondido; 3 pontos para procura do objeto considerando os deslocamentos visíveis; 4 pontos para procura do objeto considerando os deslocamentos invisíveis.

Visando garantir maior fidedignidade e confiança dos resultados, dois juízes, alunos de pós-graduação em nível de mestrado e doutorado, com vários anos de experiência no trabalho com crianças pequenas e na utilização da metodologia empregada neste estudo, fizeram a reanálise de $20 \%$ das fitas ${ }^{43,44}$.

A presente pesquisa foi aprovada pelo Conselho de Ética e Pesquisa do Hospital Universitário (CEPHU) sob número 692/05, e o Termo de Consentimento Livre Pós-Esclarecido foi assinado pelos pais ou responsável.

Os dados foram analisados de forma quantitativa e qualitativa. Para o estudo estatístico foram realizados os testes de Mann Whitney, KruskalWallis e o de Igualdade de Duas Proporções. Para o presente estudo, foi considerado um nível de significância de $5 \%$, sendo todos os intervalos de confiança construídos com $95 \%$ de confiança estatística.

\section{RESULTADOS}

As Tabelas 1 e 2 mostram a divisão da amostra segundo sexo, média da idade gestacional e peso ao nascimento para cada grupo.

A análise quantitativa evidenciou diferenças es- 
tatisticamente significantes entre o GP e GC em relação a todas as etapas da construção da noção de permanência do objeto: não procura o objeto escondido ( $p<0,001)$, procura o objeto parcialmente escondido $(p=0,03)$, procura o objeto totalmente escondido ( $p=0,021)$, procura o objeto considerando os deslocamentos visíveis $(p<$ 0,001 ) e procura o objeto considerando os deslocamentos invisíveis $(p<0,001)$. A última etapa do processo de construção da permanência do objeto (procura do objeto considerando os deslocamentos invisíveis) foi alcançada por 3 crianças $(27,3 \%)$ do GC aos 10 meses e pelas demais ( 8 crianças; $72,7 \%$ ) aos 11 meses de idade, enquanto que o GP ( $100 \%$ das crianças, $n=8)$ atingiu apenas a penúltima etapa deste processo (procura do objeto considerando apenas os deslocamen- tos visíveis) aos 12 meses de idade corrigida. Essa diferença também foi observada na análise qualitativa, a qual mostrou que o GP persistiu por um período de tempo maior em algumas etapas deste processo. Tais resultados podem ser melhor visualizados nas Figuras 1 e 2.

Considerando os resultados para cada faixa etária, observou-se diferença estatisticamente significante entre os grupos para todas as faixas de idade, evidenciando o atraso no desenvolvimento cognitivo, durante o período sensório-motor, apresentado pelo GP, em relação ao GC. Estes dados podem ser melhor visualizados na Tabela 3.

A análise de concordância revelou $96,5 \%$ de concordância entre a análise dos dados realizada pelo pesquisador e a reanálise de $20 \%$ das fitas realizada pelos juízes.

\section{Tabela 1 - Caracterização da amostra segundo o sexo}

\begin{tabular}{ccc}
\hline & \multicolumn{2}{c}{ Genêro } \\
\hline GC & $43 \%$ & Masculino \\
GP & $25 \%$ & $75 \%$ \\
\hline
\end{tabular}

Tabela 2 - Caracterização da amostra de acordo com a idade gestacional e peso médio ao nascimento

\begin{tabular}{ccccc}
\hline & Idade Gestacional* $^{*}$ & Desvio Padrão & Peso $(\mathrm{g})$ & Desvio Padrão \\
\hline GC & 39 & 1 & 3267 & 324 \\
GP & $291 / 7$ & 2,9 & 986 & 283 \\
\hline
\end{tabular}

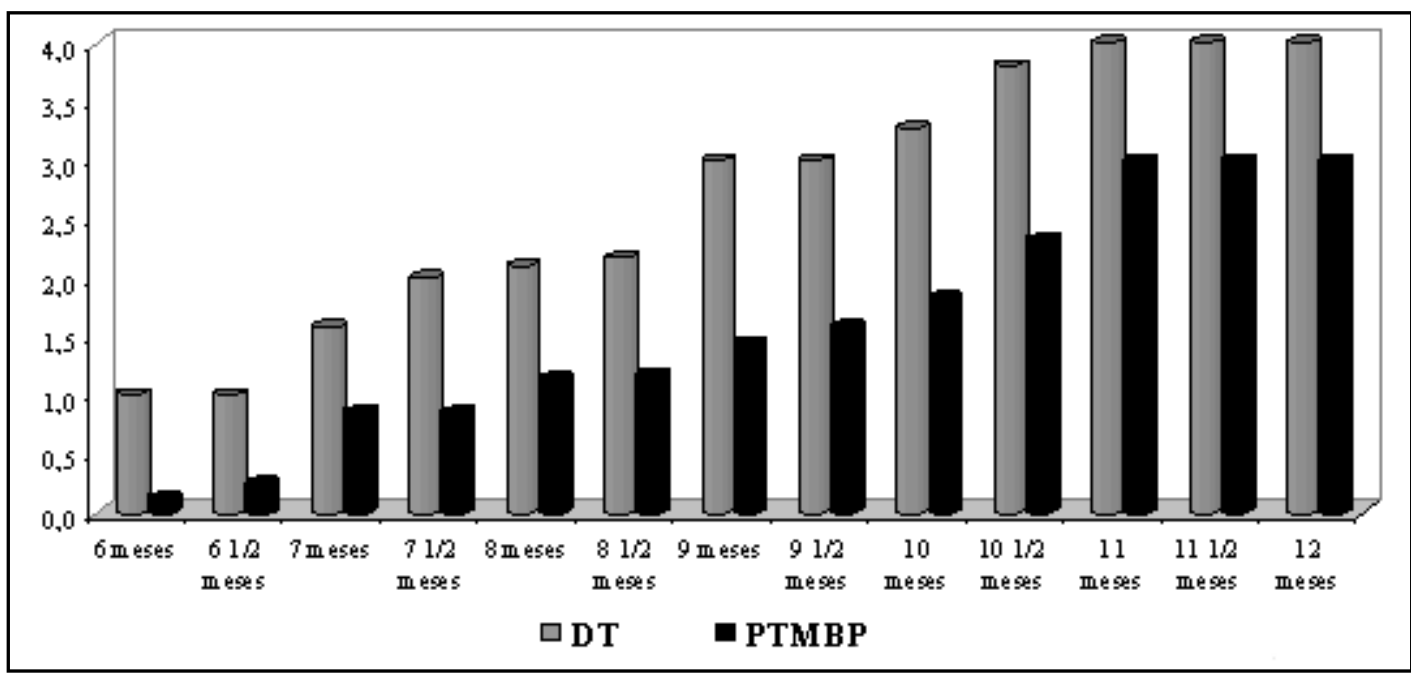

Figura 1 - Comparação entre os resultados obtidos para crianças nascidas Pré-Termo Muito Baixo Peso (PTMBP) em idade corrigida e crianças com desenvolvimento típico (DT) para cada faixa etária 


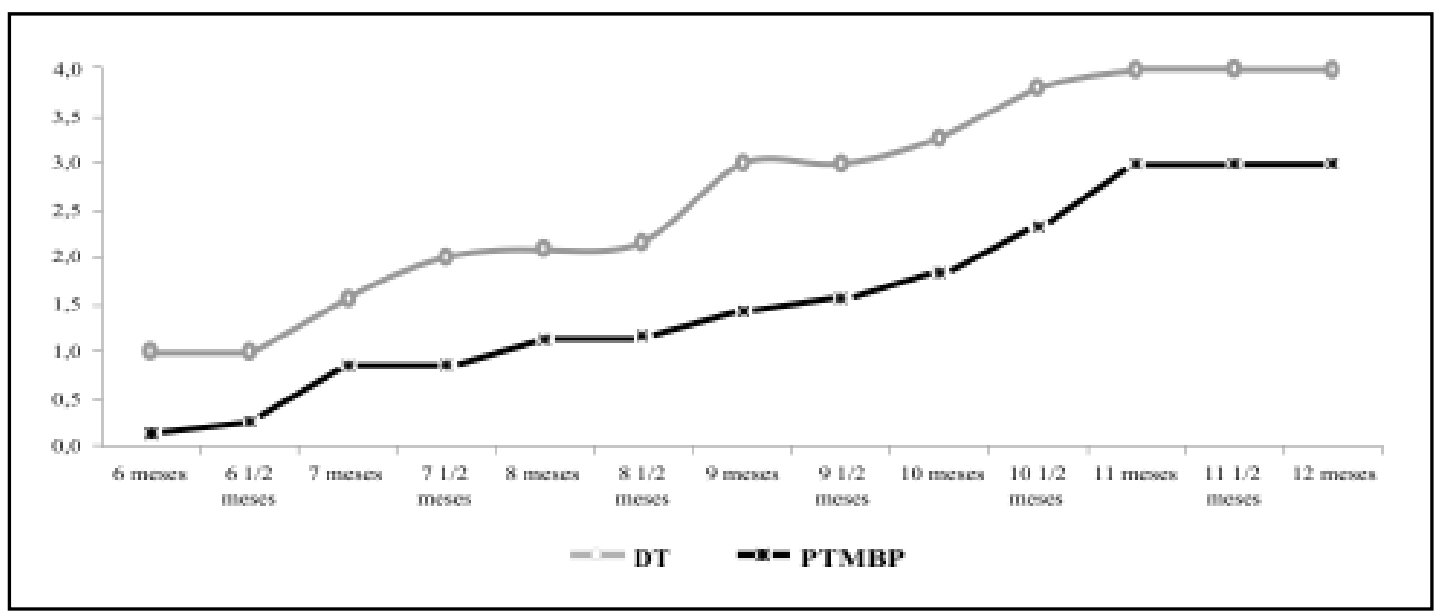

Figura 2 - Comparação da evolução da construção da permanência do objeto entre crianças nascidas Pré-Termo Muito Baixo Peso (PTMBP) em idade corrigida e crianças com desenvolvimento típico (DT)

Tabela 3 - Comparação entre a pontuação obtida para crianças nascidas PTMBP em idade corrigida e crianças com DT para cada faixa etária

\begin{tabular}{|c|c|c|c|c|c|c|c|c|c|}
\hline Idade & Grupo & Média & Mediana & $D P$ & Q1 & Q3 & TA & IC & p-valor \\
\hline \multirow[t]{2}{*}{$6 m$} & $G C$ & 1,00 & 1 & 0,00 & 1 & 1 & 8 & $-x$. & \multirow{2}{*}{$0,001^{*}$} \\
\hline & GP & 0,13 & 0 & 0,35 & 0 & 0 & 8 & 0,24 & \\
\hline \multirow[t]{2}{*}{$6,5 \mathrm{~m}$} & $G C$ & 1,00 & 1 & 0,00 & 1 & 1 & 7 & $-x-$ & \multirow{2}{*}{$0,004^{*}$} \\
\hline & GP & 0,25 & 0 & 0,46 & 0 & 0,25 & 8 & 0,32 & \\
\hline \multirow[t]{2}{*}{$7 \mathrm{~m}$} & $G C$ & 1.57 & 2 & 0,55 & 1 & 2 & 7 & 0,41 & \multirow{2}{*}{$0,019^{*}$} \\
\hline & GP & 0,86 & 1 & 0,38 & 1 & 1 & 7 & 0,28 & \\
\hline \multirow[t]{2}{*}{$7,5 \mathrm{~m}$} & $\mathrm{GC}$ & 2,00 & 2 & 0,00 & 2 & 2 & 5 & $-x-$ & \multirow{2}{*}{$0,002^{*}$} \\
\hline & GP & 0,86 & 1 & 0,38 & 1 & 1 & 7 & 0,28 & \\
\hline \multirow[t]{2}{*}{$8 m$} & GC & 2,10 & 2 & 0,00 & 2 & 2 & 10 & $-x=$ & \multirow{2}{*}{$<0,001^{\circ}$} \\
\hline & GP & 1,13 & 1 & 0,35 & 1 & 1 & 8 & 0,24 & \\
\hline \multirow[t]{2}{*}{$8,5 \mathrm{~m}$} & $\mathrm{GC}$ & 2,17 & 2 & 0,00 & 2 & 2 & 6 & $-x=$ & \multirow{2}{*}{$0,006^{*}$} \\
\hline & GP & 1,17 & 1 & 0,41 & 1 & 1 & 6 & 0,33 & \\
\hline \multirow[t]{2}{*}{$9 m$} & $\mathrm{GC}$ & 3,00 & 3 & 0,00 & 3 & 3 & 9 & $-x-$ & \multirow{2}{*}{$<0,001^{\circ}$} \\
\hline & GP & 1,43 & 1 & 0,53 & 1 & 2 & 7 & 0,40 & \\
\hline \multirow[t]{2}{*}{$9,5 \mathrm{~m}$} & $G C$ & 3,00 & 3 & 0,00 & 3 & 3 & 9 & $-x-$ & \multirow{2}{*}{$<0,001^{\circ}$} \\
\hline & GP & 1.57 & 2 & 0,53 & 1 & 2 & 7 & 0,40 & \\
\hline \multirow[t]{2}{*}{$10 \mathrm{~m}$} & $\mathrm{GC}$ & 3,27 & 3 & 0,48 & 3 & 3.5 & 11 & 0,29 & \multirow{2}{*}{$0,001^{*}$} \\
\hline & GP & 1.83 & 2 & 0,75 & 1,25 & 2 & 6 & 0,60 & \\
\hline \multirow[t]{2}{*}{$10,5 \mathrm{~m}$} & GC & 3,80 & 4 & 0,45 & 4 & 4 & 5 & 0,39 & \multirow{2}{*}{$0,007^{*}$} \\
\hline & GP & 2,33 & 2 & 0,52 & 2 & 2,75 & 6 & 0,41 & \\
\hline \multirow[t]{2}{*}{$11 \mathrm{~m}$} & GC & 4,00 & 4 & 0,00 & 4 & 4 & 12 & $-x=$ & \multirow{2}{*}{$<0,001^{\circ}$} \\
\hline & GP & 3,00 & 3 & 0,00 & 3 & 3 & 7 & $-x=$ & \\
\hline \multirow[t]{2}{*}{$11,5 \mathrm{~m}$} & $\mathrm{GC}$ & 4,00 & 4 & 0,00 & 4 & 4 & 14 & $-x-$ & \multirow{2}{*}{$<0,001^{\circ}$} \\
\hline & GP & 3,00 & 3 & 0,00 & 3 & 3 & 7 & $-x-$ & \\
\hline \multirow[t]{2}{*}{$12 \mathrm{~m}$} & GC & 4,00 & 4 & 0,00 & 4 & 4 & 14 & $-x-$ & \multirow{2}{*}{$<0,001^{\circ}$} \\
\hline & GP & 3,00 & 3 & 0,00 & 3 & 3 & 8 & $-x-$ & \\
\hline
\end{tabular}

GC - Grupo Controle; GP - Grupo Pesquisa; DP - Desvio Padrão; Q1 - Quartil 1; Q3 - Quartil 3; TA - Tamanho Amostral; IC - Intervalo de Confiança; Teste Estatístico: Teste não paramétrico de Mann-Whitney 


\section{DISCUSSÃO}

A literatura tem apontado para a presença de alterações e/ou atrasos em relação ao desenvolvimento cognitivo e de linguagem em crianças com histórico de prematuridade e muito baixo peso ${ }^{12,25-27}$. Grande parte dos estudos nessa área refere-se a crianças em idade escolar ou pré-escolar ${ }^{4-7,12,15,17-23,25,30,31}$. No presente estudo, buscou-se comprovar que é possível detectar tais alterações ou atrasos já nos primeiros anos de vida, sendo possível intervir precocemente, buscando-se prevenir ou minimizar futuras alterações decorrentes. Para tanto, foi proposto o estudo do processo de construção da noção de permanência do objeto, por se tratar de construção cognitiva fundamental, visto que leva à formação da imagem mental e à capacidade de representação, condições essenciais ao desenvolvimento da linguagem ${ }^{35-37}$.

Os resultados revelaram atraso significativo das crianças do GP em relação às crianças do GC. Observouse, também, que os prematuros extremos (2 crianças), nascidos com menos de $750 \mathrm{~g}$, apresentaram maior atraso no processo de construção da noção de permanência do objeto, persistindo por um período de tempo prolongado (dos 7 aos 10 meses de idade corrigida) na primeira etapa desse processo (procura do objeto parcialmente escondido). Estes dados corroboram outros estudos que apontam as crianças nascidas PTMBP como uma população de risco para alterações e/ou atrasos no desenvolvimento cognitivo e de linguagem ${ }^{4-13,22-28}$, e que tais alterações ou atrasos são mais significativos quanto mais baixo for o peso ao nascimento ${ }^{7,13,30-32}$.

Os procedimentos adotados neste estudo mostraram-se eficientes e permitiram a caracterização do processo de construção da noção de permanência do objeto pelos sujeitos desta pesquisa, evidenciando diferenças importantes na comparação entre os grupos. Todas as crianças seguiram as mesmas etapas de construção da noção de permanência do objeto, porém, as crianças do GP persistiram em uma mesma etapa por um período de tempo maior. Assim, as crianças do GC apresentaram procura do objeto parcialmente escondido até os
7 meses, enquanto as crianças do GP persistiram nesta etapa até os 9 meses e meio, com exceção dos prematuros extremos que persistiram até os 10 meses de idade corrigida. Da mesma forma, as crianças do GC apresentaram procura do objeto considerando os deslocamentos invisíveis (etapa final do processo) a partir dos 10 meses, enquanto que as crianças do GP não alcançaram esta etapa ao final do estudo, ou seja, aos 12 meses de idade corrigida. Evidencia-se, assim, um atraso médio de 2 a 3 meses em relação às crianças do GC, média semelhante à encontrada em outro estudo ${ }^{11}$.

Durante a aplicação das provas, observou-se que as crianças do GP apresentaram-se mais dispersas, não sendo capazes de prestar atenção na atividade por muito tempo, interessando-se a todo o momento por outros estímulos e objetos presentes na sala. Em função de tal comportamento, foi necessário chamar a atenção da criança diversas vezes, modificando os objetos que seriam escondidos sob os lenços. Tal comportamento pode, também, estar relacionado ao atraso observado em relação à construção da noção de permanência do objeto, visto que a atenção é habilidade fundamental nesse processo. Outros estudos também apontam para presença de dificuldades ou déficits de atenção, entre outras disfunções neurológicas ${ }^{17-20,29}$, estimando que ocorram em $50 \%$ a $70 \%$ das crianças nascidas PTMBP ${ }^{21}$.

\section{CONCLUSÃO}

As diferenças encontradas entre as crianças nascidas PTMBP e as crianças com desenvolvimento típico, em relação ao processo de construção da noção de permanência do objeto, evidenciam atraso cognitivo das crianças nascidas PTMBP em idade corrigida. Tais resultados justificam não apenas o acompanhamento dos recém-nascidos PTMBP, mas a elaboração de um programa de intervenção fonoaudiológica, desde o período sensório-motor, visando minimizar tais riscos contribuindo, assim, para uma melhor qualidade de vida dessas crianças.

\section{ABSTRACT}

Purpose: to describe and compare the process of object permanence construction in very low birth weight preterm and full-term typical developing infants. Methods: eight very low birth weight infants (experimental group-EG) and 14 full-term infants (control group-CG) were monthly evaluated as for language and cognition abilities, including the assessment of the object permanence notion. All sessions were recorded in video and transcribed in specific protocols. Children of both groups were evaluated from 6 to 12 months of age, considering the corrected gestational age for the EG. Quantitative and qualitative analysis were carried out. Results: we found statistically significant differences between EG and CG concerning all the stages of object permanence development, evidencing cognitive delays (about 2 or 3 months delay) for the EG, when compared with the CG, during the sensorimotor period. Conclusions: the findings of this study corroborate with the idea that very low birth weight infants are considered at risk for language and cognition deficits or impairments, justifying the elaboration of early intervention programs during the first year of life.

KEYWORDS: Child Development; Cognition; Premature; Infant 


\section{REFERÊNCIAS}

1. Perlman JM. Neurobehavioral deficits in premature graduates of intensive care potential medical and neonatal environmental risk factors. Pediatrics. 2001; 108(6):1339-48.

2. Rugolo LM. Crescimento e desenvolvimento a longo prazo do prematuro extremo. J Pediatr. 2005; 81:S101-10.

3. Mello RR, Dutra MVP, Silva KS, Lopes JMA. Valores de predição da avaliação neurológica e ultrasonográfica cerebral neonatal em relação ao desenvolvimento de prematuros de muito baixo peso. Rev Saúde Pública. 1998; 32(5):420-9.

4. Luoma L, Herrgard E, Martikainen A, Ahonen T. Speech and language development of children born at $<$ or $=32$ week's gestation: a 5-year prospective followup study. Dev Med Child Neurol. 1998; 40(6):380-7. 5 . Wolke D, Meyer R. Cognitive status, language attainment, and prereading skills of 6-year-old very preterm children and their peers: the Bavarian Longitudinal Study. Dev Med Child Neurol. 1999; 41(2):94-109. 6. Anderson P, Doyle LW. Neurobehavioral outcomes of school-age children born extremely low birth weight or very preterm in the 1990s. JAMA. 2003; 289(24):3264-72.

7. Taylor HG, Minich NM, Klein N, Hack M. Longitudinal outcomes of very low birth weight: neuropsychological findings. J Int Neuropsychol Soc. $2004 ; 10(2): 149-63$.

8. Briscoe J, Gathercole SE, Marlow N. Short-term memory and language outcomes after extreme prematurity at birth. J Speech Lang Hear Res. 1998; 41(3):654-66.

9. Peterson BS, Vohr B, Kane MJ, Whalen DH, Schneider KC, Katz KH, Zhang H, Duncan CC, Makuch R, Gore JC, Ment LR. A functional magnetic resonance imaging study of language processing and its cognitive correlates in prematurely born children. Pediatrics. 2002; 110(6):1153-62.

10. Jansson-Verkasalo E, Ceponiene R, Valkama M, Vainionpää L, Laitakari K, Alku P, Suominen K, Näätänen R. Deficient speech-sound processing, as shown by the electrophysiologic brain mismatch negativity response, and namein ability in prematurely born children. Neurosci Lett. 2003; 348(1):5-8.

11. Cusson RM. Factors influencing language development in preterm infants. J Obstet Gynecol Neonatal Nurs. 2003; 32(3):402-9.

12. Caravale B, Tozzi C, Albino G, Vicari S. Cognitive development in low risk preterm infants at 3-4 years of life. Arch Dis Child Fetal Neonatal Ed. 2005; 90(6):F474- 9.

13. Sansavini A, Guarini A, Alessandroni R, Faldella G, Giovanelli G, Salvioli G. Early relations between lexical and grammatical development in very immature Italian preterms. J Child Lang. 2006; 33(1):199-216.
14. Pedromônico MRM, Azevedo MF, Kopelman BI. Recém-nascidos pré-termo internados em unidade de terapia intensiva: desenvolvimento da conduta interativa no primeiro ano de vida. J Pediatr. 1998; 74:284-90.

15. Diniz CF, Pedromônico MRM, Perissinoto J. Prematuridade: intercorrências clínicas perinatais e resultados de avaliações fonoaudiológicas. In: Sociedade Brasileira de Fonoaudiologia, organizador. Atualização em voz, linguagem, audição e motricidade oral. São Paulo: Frôntis; 1999. p. 345-59.

16. Chen PS, Jeng SF, Tsou KI. Developmental function of very-low-birth-weigth infants and full-term infants in early childhood. J Formos Med Assoc. 2004; 103(1): 23-31.

17. Carvalho AEV, Linhares MBM, Martinez FE. História de desenvolvimento e comportamento de crianças nascidas pré-termo e baixo peso $(<1.500 \mathrm{~g})$. Psicol Reflex Crít. 2001; 14(1):1-33.

18. Hack M, Flannery DJ, Schluchter M, Cartar L, Borawski E, Klein N. Outcomes in young adulthood for very-low-birth-weight infants. N Engl J Med. 2002; 346(3):149-57.

19. Hansen BM, Dinesen J, Hoff B, Greisen G. Intelligence in preterm children at four years of age as a predictor of school function: a longitudinal controlled study. Dev Med Child Neurol. 2002; 44(8):517-21.

20. Foulder-Hughes LA, Cooke RWI. Motor, cognitive and behavioral disorders in children born very preterm. Dev Med Child Neurol. 2003; 45:97-103.

21. Taylor HG, Klein N, Minich NM, Hack M. Middleschool-age outcomes in children with very low birthweight. Child Dev. 2000; 71(6):1495-511.

22. Horwood LI, Magridge N, Darlow BA. Cognitive, educational and behavioral outcomes at 7 to 8 years in a national very low birth weight cohort. Arch Dis Child Fetal Neonatal. 1998; 79:2-20.

23. Jennische $M$, Sedin G. Linguistic skills at $6 \frac{1}{2}$ years of age in children who required neonatal intensive care in 1986-1989. Acta Pediatr. 2001; 90(2):199-212.

24. Oliveira LN, Lima MCMP, Gonçalves VMG. Acompanhamento de lactentes com baixo peso ao nascimento: aquisição de linguagem. Arq Neuropsiquiatr. 2003; 61(3-B):802-7.

25. Ishii C, Miranda CS, Isotani SM, Perissinoto J. Caracterização de comportamentos lingüísticos de crianças nascidas prematuras, aos quatro anos de idade. Rev CEFAC. 2006; 8(2):147-54.

26. Haan M, Bauer PJ, Georgieff MK, Nelson CA. Explicit memory in low-risk infant aged 19 months born between 27 e 42 week of gestation. Dev Med Child Neurol. 2000; 42(5):304-12.

27. Méio MDBB, Lopes CS, Morsch DS. Fatores prognósticos para o desenvolvimento cognitivo de prematuros de muito baixo peso. Rev Saúde Pública. 2003; $37(3): 311-8$. 
28. Kilbride HW, Thorstad K, Daily DK. Preschool outcome of less than 801-gram preterm infants compared with full-term siblings. Pediatrics. 2004; 113(4):742-7.

29. Vicari S, Caravale B, Carlesimo GA, Casadei AM, Allemand F. Spatial memory deficits in children at ages 3-4 who were low birth weight, preterm infants. Neuropsychology. 2004; 18(4):673-8.

30. Msall ME, Tremont MR. Functional outcomes in self-care, mobility, communication and learning in extremely low-birth-weight infants. Clin Perinatol. 2000; 27(2):381-401.

31. Chaudhari S, Otiv M, Chitale A, Pandit A, Hoge M. Pune low birth weight study: cognitive abilities and educational performance at twelve years. Indian Pediatr. 2004; 41(2):121-8.

32. Ozbek A, Miral S, Eminagaoglu N, Ozkan H. Development and behavior of non-handicapped preterm children from a developing contry. Pediatr Int. 2005; 47(5): 532-40.

33. StewartAL, Rifkin L, Amess PN, Kirkbride V, Townsend JP, Miller DH, Lewis SW, Kingsley DP, Moseley IF, Foster $\mathrm{O}$, Murray RM. Brain structure and neurocognitive and behavioral function in adolescents who were born very preterm. Lancet. 1999; 353(9765):1653-7.

34. Curtis WJ, Lindeke LL, Georgieff MK, Nelson CA. Neurobehavioural functioning in neonatal intensive care unit graduates in late childhood and early adolescence. Brain. 2002; 125(7):1646-59. 35. Piaget J. A formação do símbolo na criança. Rio de Janeiro: Zahar; 1978, edição original de 1946.
36. Piaget J, Inhelder B. A psicologia da criança. Rio de Janeiro: Bertrand Brasil; 1980.

37. Dongo-Montoya AO. Représentation imagée et construction de la pensée: inplications théoriques pour la pratique de la remediation cognitive. Bull Psychol. 1998; 51(5):529-36.

38. Piaget J. O nascimento da inteligência na criança. Rio de Janeiro: Zahar; 1978, edição original de 1936.

39. Piaget J. A construção do real na criança. Rio de Janeiro: Zahar; 1970, edição original de 1937. 40. Bates E, Benigni L, Bretherton I, Camaioni L, Volterra V. The emergence of symbols: cognition and communication in infancy. New York: Academic Press; 1979.

41. Iverson JM, Longobardi E, Caselli MC. Relationship between gestures and words in children with Down's syndrome and typically developing children in early stages of communicative development. Int J Languag Commun Disord. 2003; 38(2): 179-97.

42. Capone NC, McGregor KK. Gesture development: a review for clinical and research practices. J Speech Lang Hear Res. 2004; 47(1):173-86.

43. Moore MK, Meltzoff NA. Object permanence after a $24 \mathrm{~h}$ delay and leaving the locale of disapperance: the role of memory, space and identity. Dev Psychol. 2004; 40(4):606-20.

44. Ruffman T, Slade L, Redman J. Young infants' expectations about hidden objects. Cognition. 2005; 97(2):B35-43.

\section{RECEBIDO EM: 01/03/2007 \\ ACEITO EM: 24/07/2007}

Endereço para correspondência:

Rua Cipotânia, 51

São Paulo - SP

CEP: $05360-160$

Tel: (11) 30917455 / Fax: (11) 30917714

E-mail: kbuhler@hu.usp.br 